\title{
Nerolidol Attenuates Oxidative Stress, Inflammation, and Apoptosis by Modulating Nrf2/MAPK Signaling Pathways in Doxorubicin-Induced Acute Cardiotoxicity in Rats
}

\author{
Seenipandi Arunachalam ${ }^{1}$, M. F. Nagoor Meeran ${ }^{1}$ (D), Sheikh Azimullah ${ }^{1}$ (D) Charu Sharma $^{2}$, Sameer N. Goyal ${ }^{3}$ \\ and Shreesh Ojha $1, * \mathbb{D}$ \\ 1 Department of Pharmacology and Therapeutics, College of Medicine and Health Sciences, \\ United Arab Emirates University, Al Ain 17666, United Arab Emirates; seenipandi@uaeu.ac.ae (S.A.); \\ nagoormeeran1985@uaeu.ac.ae (M.F.N.M.); azim.sheikh@uaeu.ac.ae (S.A.) \\ 2 Department of Internal Medicine, College of Medicine and Health Sciences, United Arab Emirates University, \\ Al Ain 17666, United Arab Emirates; charusharma@uaeu.ac.ae \\ 3 Shri Vile Parle Kelavani Mandal's Institute of Pharmacy, Dhule 424001, India; goyal.aiims@gmail.com \\ * Correspondence: shreeshojha@uaeu.ac.ae; Tel.: +971-3713-7524
}

\section{check for} updates

Citation: Arunachalam, S.; Nagoor Meeran, M.F.; Azimullah, S.; Sharma, C.; Goyal, S.N.; Ojha, S. Nerolidol Attenuates Oxidative Stress,

Inflammation, and Apoptosis by Modulating Nrf2/MAPK Signaling Pathways in Doxorubicin-Induced Acute Cardiotoxicity in Rats. Antioxidants 2021, 10, 984. https:// doi.org/10.3390/antiox10060984

Academic Editor: Tommaso Angelone

Received: 8 April 2021

Accepted: 13 May 2021

Published: 21 June 2021

Publisher's Note: MDPI stays neutral with regard to jurisdictional claims in published maps and institutional affiliations.

Copyright: (c) 2021 by the authors. Licensee MDPI, Basel, Switzerland. This article is an open access article distributed under the terms and conditions of the Creative Commons Attribution (CC BY) license (https:// creativecommons.org/licenses/by/ $4.0 /$ )

\begin{abstract}
The clinical usage of doxorubicin (DOX), a potent anthracycline antineoplastic drug, is often limited by its cardiotoxic effects. Thus, for improving usage of DOX, the aim of this study was to assess the cardioprotective effects of nerolidol (NERO) in a rat model of DOX-induced acute cardiotoxicity and examine underlying molecular mechanisms that contribute to these effects. To induce acute cardiotoxicity male albino Wistar rats were injected with single dose intraperitoneal DOX $(12.5 \mathrm{mg} / \mathrm{kg})$. The rats were treated with NERO $(50 \mathrm{mg} / \mathrm{kg}$, orally) for five days. DOX-injected rats showed elevated levels of cardiac marker enzymes and enhanced oxidative stress markers along with altered Nrf2/Keap1/HO-1 signaling pathways. DOX administration also induced the activation of NF- $\mathrm{B} / \mathrm{MAPK}$ signaling and increased the levels and expression of pro-inflammatory cytokines (TNF- $\alpha$, IL-6, and IL-1 $\beta$ ) as well as expression of inflammatory mediators (iNOS and COX-2) in the heart. DOX also triggered DNA damage and apoptotic cell death in the myocardium. Additionally, histological studies revealed structural alterations of the myocardium. NERO treatment exhibited protection against the deleterious results of DOX on myocardium, as evidenced by the restoration of altered biochemical parameters, mitigated oxidative stress, inflammation, and apoptosis. The findings of the present study demonstrate that NERO provides cardioprotective effects against DOXinduced acute cardiotoxicity attributed to its potent antioxidant, anti-inflammatory, and antiapoptotic activities through modulating cellular signaling pathways.
\end{abstract}

Keywords: acute cardiotoxicity; cardioprotective; doxorubicin; inflammation; nerolidol; oxidative stress; sesquiterpene

\section{Introduction}

Chemotherapeutic agents have significantly improved the likelihood of survival among cancer patients [1]. Of the numerous chemotherapeutic agents, doxorubicin (DOX) also known as Adriamycin belongs to anthracycline class of cytotoxic antibiotic, which is extensively used in the treatment of multiple types of malignancies sarcoma, carcinoma, lymphoma and leukemia [2]. However, the clinical use of DOX is often limited due to its severe adverse effects, including neurological disturbances, bone marrow aplasia and cardiotoxicity [3]. The appearance of acute cardiotoxicity necessitated the need for effective agents to mitigate DOX-induced acute cardiotoxicity [4].

To reduce the occurrence of acute cardiotoxicity induced by DOX, numerous protective strategies have been developed. This includes the development of improved dosage forms of DOX itself, as well as the development of agents that mitigate oxidative stress, inflammation, and apoptosis. This leads to biochemical, molecular, structural and histological 
alterations and appear as common pathogenic events in DOX-induced acute cardiotoxicity $[5,6]$. Numerous antioxidants, anti-inflammatory and antiapoptotic agents of natural and synthetic origins, have been shown to exert protective effects in preclinical models of DOX-induced cardiotoxicity. Among numerous synthetic antioxidants, dexrazoxane, a derivative of the chelating agent ethylenediaminetetra-acetic acid appears one of the promising agents for the treatment of DOX-induced cardiotoxicity. Though, synthetic antioxidant, dexrazoxane has been found potentially beneficial in reducing the generation of oxygen radical species [7]. But, it's like other synthetic antioxidants possesses adverse effects. Thus, many naturally occurring plant-derived secondary metabolites, termed phytochemicals, have received enormous attention for their potential chemopreventive, chemotherapeutic, and cardioprotective benefits [8-10].

In recent years, numerous phytochemicals have been showed to exert chemopreventive, chemotherapeutic, chemosensitizing, and cardioprotective properties that mediate between antioxidant and anti-inflammatory properties, along with cell death pathway modulation [8-11]. Accumulative studies over the years demonstrated that numerous mechanisms are involved in DOX-induced cardiotoxicity. One of the important mechanisms is the overproduction of free radicals, which leads to the exhaustion of endogenous antioxidant defense, lipid peroxidation, induction of inflammatory mediators, DNA damage, programmed cell death, and necrosis in an orchestrated manner [12,13]. DOX has been also shown to elicit a significant rise in the levels of pro-inflammatory cytokines and inflammatory mediators in the myocardium [14]. Nuclear factor kappa-B (NF-kB) activation and mitogen-activated protein (MAPK) signaling is known to trigger apoptosis by activating a pro-apoptotic event [15]. DOX also showed to induce apoptosis via c-Jun $\mathrm{N}$-terminal kinases (JNKs) and MAP kinase signaling pathways [16].

Additionally, NF- $\mathrm{KB}$ inversely regulates the transcription and activities of nuclear factor erythroid 2-related factor 2 (Nrf2), a transcription factor. Nrf2, a master regulator of oxidative stress, is known to orchestrate redox defense mechanisms in the event of oxidative stress via the augmentation of endogenous antioxidant defense mechanisms and the activation of hemeoxygenase- 1 (HO-1) $[17,18]$. Nrf2 and its principal negative regulator, the E3 ligase adaptor Kelch-like ECH-associated protein 1 (Keap1), potentially appears to be a therapeutic target to mitigate DOX-induced cardiotoxicity through sustaining antioxidant defense mechanisms at cellular levels in the myocardial cells. To curb the multifactorial nature of DOX-induced cardiotoxicity, the naturally occurring agents appears to mitigate oxidative stress, inflammation, and apoptosis, which could be important for mitigating pathogenic events of oxidative stress, inflammation and apoptosis [19]. Many antioxidant, anti-inflammatory and antiapoptotic agents have been shown to improve endogenous antioxidants, as well as reduce inflammatory and apoptotic cascades by promoting cell survival and cell signaling pathways [19].

In recent years, many naturally occurring phytochemicals have been revealed to mitigate DOX-induced cardiotoxicity [20-22]. Among numerous phytochemical classes, terpenes and terpenoids have garnered attention for their potential health benefits and therapeutic and preventive potential in cancers and cardiovascular diseases $[9,10]$. One of the sesquiterpenes, nerolidol (NERO), has specifically received interest for its potential benefits in cancers and cardiovascular diseases [23]. NERO is abundantly found in the essential oils of many ornamental, medicinal, and edible or dietary plants [23]. It is one of the common components of Oolong tea (a traditional semi-oxidized Chinese tea) and is known to contribute to the floral aroma to tea, the world's most popular beverage [24]. It is also present in Madeira wines from Portugal [25]. It is characterized with a floral odor and contribute to flavor of fruits including kiwis and strawberries. It is used as a flavor enhancer in foods, cosmetics, and beverages [26,27].

NERO is approved by the Food and Drug Administration (FDA) and listed in catalogue of substances "generally recognized as safe" (GRAS) for use in food and beverages [23]. NERO also possesses antimicrobial, antibiofilm, antioxidant, antiparasitic, anti-nociceptive, anti-inflammatory, and anticancer properties $[23,28]$. It has been shown 
to activate antioxidant signaling pathways and attenuate inflammatory and apoptotic signaling pathways, as well as exert cytoprotective properties by improving antioxidant defense [29] and exerting anti-inflammatory effects [23,30,31]. Recently, NERO has been shown to attenuate oxidative stress, inflammation, fibrosis, and apoptosis in cardiotoxicity induced by cyclophosphamide, a chemotherapeutic agent [32], isoproterenol-induced myocardial infarction [33], and modulate contractile function of the atrium [34]. Additionally, NERO has been shown effective as an anticancer agent due to its potential to modulate important molecular targets of cell survival and proliferation [35], as well as act as a chemosensitizer in cancer cells [36-38]. NERO has also been shown to enhance the efficacy of DOX in breast cancer cells [36] and improve DOX efficacy in sensitive and partly resistant lymphoblast and ovarian cancer cells [39].

NERO showed to improve DOX accumulation in cancer cells without affecting DOX concentration in hepatocytes [37]. It also showed to exert an inhibitory effect on carcinogenesis of the large intestine [38]. The potent cardioprotective, chemopreventive, anticancer, and chemosensitizing property of NERO needs to be further evaluated for its potential in DOX-induced acute cardiotoxicity given the preclinical evidence that it synergistically interacts with DOX in cancer chemotherapy. The aim of this study was to evaluate the cardioprotective effect of NERO in acute cardiotoxicity. Our findings further substantiate the fact that DOX is used more than cyclophosphamide in a wide variety of cancers. Moreover, NERO was found not to affect the clinical efficacy of DOX, as it exerted synergistic effects on resistant cancer cells [36-39].

In view of this, it is important to evaluate the cardioprotective potential of NERO against DOX-induced acute cardiotoxicity in rats. More specifically, to better understand the role of NERO in DOX-induced acute cardiotoxicity, we investigated the effect of NERO on antioxidant defense, inflammatory markers, and apoptosis. As such, we elucidated the molecular mechanisms of cardioprotection attributed by the modulation of cell signaling pathways.

\section{Materials and Methods}

\subsection{Drugs, Chemicals and Kits}

The test compound, Nerolidol was purchased from Sigma Aldrich, St. Louis, MO, USA. Doxorubicin was purchased from EBEWE Pharma, Unterach, Mondseestrasse, Austria. The commercially available kits for the biochemical estimations were obtained from Sigma Aldrich, St. Louis, MO, USA and Abcam, MA, USA.

\subsection{Experimental Animals}

The animal research protocols and procedures were approved by the United Arab Emirates University Animal Ethics Committee (ERA_2019_5919). Male adult albino Wistar rats in the weight range of 180-190 g were obtained from the University Animal Research Facility at the College of Medicine and Health Sciences (CMHS), United Arab Emirates University, UAE. The animals were kept in polypropylene animal cages $(47 \times 34 \times 20 \mathrm{~cm})$, which contained a layer of husk that was replaced every $24 \mathrm{~h}$. The animal facility maintained at standard animal housekeeping, including a photoperiod of $12 \mathrm{~h} \mathrm{light/dark} \mathrm{cycle,}$ a temperature of $22{ }^{\circ} \mathrm{C}$, and a humidity of $45-55 \%$. The animals were fed with a standard rodent chow diet procured from the National Feed and Flour Production and Marketing Company, LLC., Abu Dhabi, UAE. The animals had free access to water ad libitum.

\subsection{Induction of Cardiotoxicity in Rats}

To induce acute cardiotoxicity in rats, a single dose of DOX $(12.5 \mathrm{mg} / \mathrm{kg}$ body weight) was intraperitoneally injected [6]. The changes in the levels of myocardial enzymes were diagnostic biomarkers of DOX-induced cardiotoxicity in rats. 


\subsection{Experimental Design}

Rats were randomly allocated into four experimental groups, each containing eight rats. Group I: normal control rats; group II: rats orally treated with NERO $(50 \mathrm{mg} / \mathrm{kg}$ body weight) and dissolved in olive oil daily for five days; group III: rats intraperitoneally injected with DOX (12.5 mg/ $\mathrm{kg}$ body weight) to induce acute cardiotoxicity; group IV: rats administered with a single intraperitoneal dose of DOX $(12.5 \mathrm{mg} / \mathrm{kg}$ body weight $)$ and orally treated with NERO (50 mg/ $\mathrm{kg}$ body weight) for five days. After the treatment period (i.e., on sixth day), all rats were anaesthetized with pentobarbital sodium $(60 \mathrm{mg} / \mathrm{kg}$ body weight) and then killed via cervical decapitation. The blood samples were collected and centrifuged at room temperature for $10 \mathrm{~min}$ at $4000 \mathrm{rpm}$. The supernatant (serum) was collected and frozen at $-80{ }^{\circ} \mathrm{C}$ until further analysis. Hearts were collected and snap frozen in liquid nitrogen before being stored at $-80^{\circ} \mathrm{C}$ for biochemical analysis. Samples were also placed in $10 \%$ neutral buffered formalin and stored at $4{ }^{\circ} \mathrm{C}$ for histological study.

\subsection{Assay of Cardiac Marker Enzymes}

The levels of serum creatine kinase (CK) and lactate dehydrogenase (LDH) was assayed using VetTest 8008 Chemistry Analyzer (UK). The levels of serum troponin-T were estimated using the commercial kit obtained from MyBiosource International, San Diego, CA, USA.

\subsection{Estimation of Lipid Peroxidation and Antioxidants}

The levels of malondialdehyde (MDA) in the heart homogenate were determined using a commercially available kit (North West Life science, WA, USA). The data obtained are expressed as $\mu \mathrm{M} / \mathrm{mg}$ protein. The concentrations of superoxide dismutase (SOD) and catalase/glutathione contents were estimated using commercially available kits following the manufacturer's instructions (Sigma Aldrich, St. Louis, MO, USA; Cayman Chemical Company, Ann Arbor, MI, USA).

\subsection{Enzyme Linked Immunosorbent Assay (ELISA)}

The levels of proinflammatory cytokines in the heart such as tumor necrosis factor- $\alpha$ (TNF- $\alpha$ ), interleukin-6 (IL-6), and interleukin-1 $\beta$ (IL-1 $\beta$ ) were measured in the myocardial homogenates using ELISA kits (MyBiosource International, San Diego, CA, USA).

\subsection{Western Blot Analysis}

Heart tissues were homogenized in a RIPA buffer with protease and phosphatase inhibitors (Sigma Aldrich, MO, USA) and the homogenates were centrifuged at 14,000 rpm ( $4{ }^{\circ} \mathrm{C}$ for $30 \mathrm{~min}$ ). The supernatant was collected and mixed well with a $4 \times$ laemmlli buffer (Bio Rad, Hercules, CA, USA) and 2-mercaptoethanol (Sigma Aldrich, St. Louis, MO, USA). By using the gel electrophoresis, equal amounts of protein were separated and transferred onto PVDF membranes (Thermo Fisher Scientific, Waltham, IL, USA). The PVDF membrane was incubated at $4{ }^{\circ} \mathrm{C}$ overnight. It contained primary antibodies against inducible nitric oxide synthase (iNOS) (anti-rabbit; Sigma Aldrich, St. Louis, MO, USA), cyclooxygenase2 (COX-2), Bcl2 associated X protein (Bax), B-cell lymphoma-2 (bcl2), B-cell lymphoma extra-large (Bcl-xL), active caspase-3, Nrf2, cytochrome-C, keap-1, HO-1, IкB- $\alpha, p$-IкB- $\alpha$, $\mathrm{t}-\mathrm{P}^{38}, p$ - $\mathrm{P}^{38}, p$-IKK $\alpha$, apoptotic protease activating factor 1 (APAF-1) (anti-rabbit and mouse, Abcam, Cambridge, MA, USA), t-IKK $\alpha$, t-Jun-amino-terminal kinase (t-JNK), p-Jun-aminoterminal kinase ( $p$-JNK), $\mathrm{p}$-67 phox, cleaved poly (ADP-ribose) polymerase (cleaved PARP) (Santa Cruz Biotechnology, Dallas, TX, USA), superoxide dismutase 1 (SOD1), superoxide dismutase 2 (SOD2), active caspase-9 (Cell Signaling Technology, Danvers, MA, USA), nuclear factor kappa-B-p65 (NF-kB-p65), phospho nuclear factor kappa-B-p65 ( $p$-NF-kBp65), and $\beta$-actin (anti-mouse; Millipore, Burlington, MA, USA). The PVDF membrane was again incubated for $1 \mathrm{~h}$ with the corresponding secondary antibodies. The protein expressions were visualized using a chemiluminescence West Pico kit (Thermo Fisher Scientific, Waltham, MA, USA). To measure the intensity of the signals, we conducted a 
densitometric analysis using Image J software (National Institutes of Health, Bethesda, MD, USA).

\subsection{Histopathological Evaluation}

Heart tissues were fixed in formalin for a week. Then, the formalin fixed tissues were processed following a series of exchanges in different solvents. Finally, they were embedded in the paraffin wax. The formalin-fixed paraffin embedded tissues were serially sectioned in 5-10 $\mu \mathrm{m}$ thickness using a microtome (Leica Biosystems, Nussloch, Germany). The tissue sections were stained with hematoxylin and eosin (H\&E). Thereafter, these were mounted on gelatin coated glass slides. The mounted sections were visualized under a light microscope BX41 obtained from Olympus, Center Valley, PA, USA and images were captured for histopathological assessment.

\subsection{Estimation of Protein Concentration}

The total amount of protein in the samples were determined using the Pierce ${ }^{\mathrm{TM}}$ BCA protein assay kit (Thermo Fisher Scientific, Waltham, IL, USA) and according to the manufacturer's instructions.

\subsection{Statistical Analysis}

The results are shown as mean \pm standard error of the mean (SEM). The differences among groups were statistically analyzed via one-way analysis of variance (ANOVA) followed by a Duncan's multiple range test (DMRT). The data were analyzed using IBM SPSS statistics v24.0 (SPSS Inc., Chicago, IL, USA). The criterion of statistical significance was set at the $p$ value less than 0.05 .

\section{Results}

3.1. Nerolidol (NERO) Reinstates Myocardial Marker Enzymes and Doxorubicin (DOX)-Induced Changes in the Myocardial Architecture in Rats

DOX-injected rats showed a significant $(p<0.05)$ increase in the serum levels of creatine kinase (CK), lactate dehydrogenase (LDH), and troponin-T compared to the normal control rats. However, treatment with NERO prevented a cardiomyocytes injury-induced release of CK, LDH, and troponin-T into the serum in DOX administered rats compared to DOX control rats. The hearts in normal and NERO treated rats showed normal myocardial histoarchitecture with no changes in the cardiomyocytes. This finding suggests that there are no adverse effects of NERO at this particular dose and that it is devoid of any adverse effects on cardiomyocytes. Rats injected with DOX showed an intense degradation of muscle fibers with clear signs of inflammation. On the other hand, DOX-injected rats treated with NERO (ND) were revealed to have reduced muscle fiber degradation with negligeable inflammatory cells (Figure 1).

\subsection{NERO Attenuates DOX-Induced Oxidative Damage via Activation of Nuclear Factor Erythroid 2-Related Factor 2 (Nrf2) Signaling Pathway}

DOX administered rats showed a significant $(p<0.05)$ increase in malondialdehyde (MDA) content with a significant $(p<0.05)$ decrease in the activities/concentrations of superoxide dismutase (SOD), catalase, and reduced glutathione (GSH), at least when compared to the normal control rats. Moreover, DOX-injected rats showed a significant $(p<0.05)$ decrease in the expressions of myocardial proteins, heme oxygenase-1 (HO-1) and Nrf2 demonstrated a significant $(p<0.05)$ increase in the expression of Kelch-like ECH-associated protein 1 (Keap-1) in the myocardium compared to the normal control rats. However, rats treated with NERO showed a significant $(p<0.05)$ reduction in MDA levels and a significant $(p<0.05)$ increase in the activities/concentration of SOD, catalase, and GSH, at least when compared to the DOX control rats. NERO treatment also showed a significant $(p<0.05)$ increase in the expressions of myocardial proteins. HO-1 and Nrf2 had a significant $(p<0.05)$ decrease in the myocardial protein expressions of Keap-1 in DOX-injected rats compared to DOX control rats (Figure 2). 
(A)

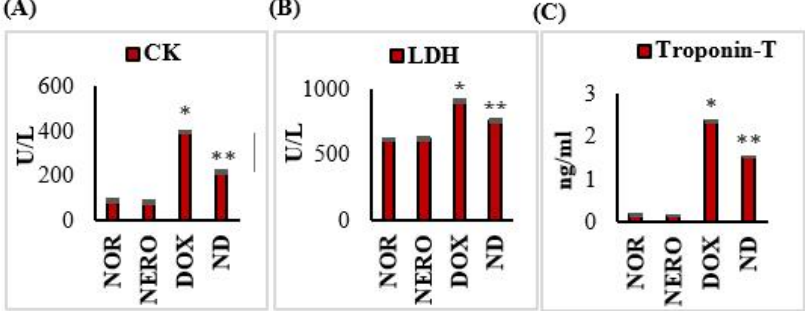

(D)

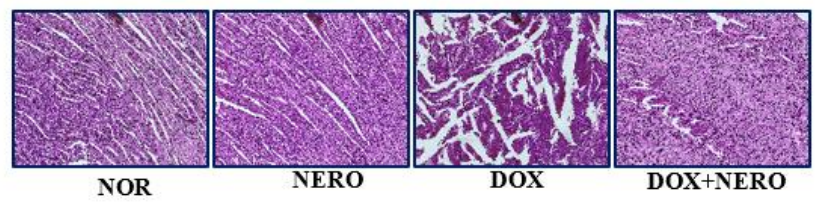

Figure 1. (A-C) Effect of NERO on myocardial injury markers; columns do not share a common symbol $(*, * *)$ and differ significantly from each other $\left({ }^{*} p<0.05\right.$ vs. normal control, ${ }^{* *} p<0.05$ vs. DOX control). (D). Histopathology of the myocardium: Normal control rat's heart has normal architecture of the myocardium; NERO alone treated rat's heart also shows normal intact muscle fibers without any pathological changes; DOX_-injected rat's heart shows extensive muscle fiber degradation with inflammatory cells; NERO treated DOX_challenged rats shows a reduced muscle fiber degradation without inflammatory cells. CK-creatine kinase; LDH-lactate dehydrogenase; NOR—normal; NERO—nerolidol; DOX—doxorubicin; ND—rats administered with a single intraperitoneal dose of DOX and orally treated with NERO.

(A)

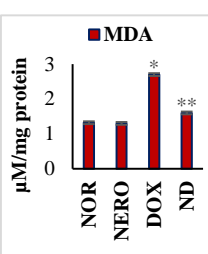

(B)

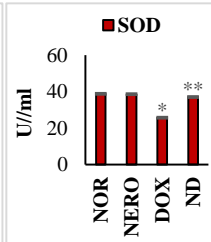

(C)

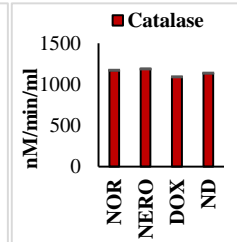

(D)

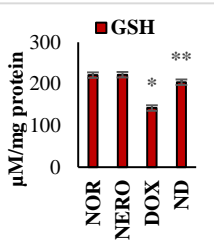

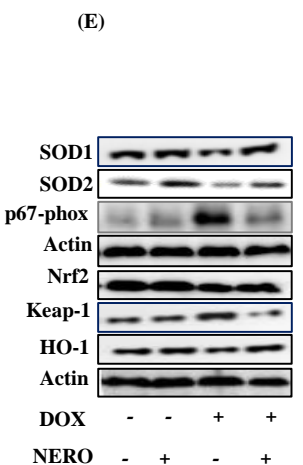

(F)
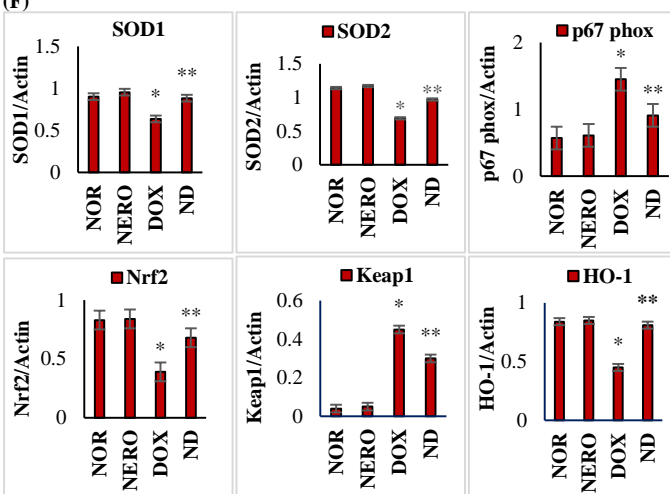

Figure 2. (A-D) Effect of NERO on lipid peroxidation and oxidative stress. Lipid peroxidation and antioxidant status in the heart. Each column is mean \pm SEM for eight rats in each group; columns do not share a common symbol $\left({ }^{*},{ }^{* *}\right)$ and differ significantly with each other $\left({ }^{*} p<0.05\right.$ vs. normal control, ${ }^{* *} p<0.05$ vs. DOX control). (E,F). Western immunoblot analysis and densitometric analysis for SOD1, SOD2, p67-phox, Nrf2, HO-1, and Keap-1. Values are expressed as mean \pm SEM $(n=3)$; columns do not share a common symbol $(*, * *)$ and differ significantly with each other ( $p<0.05$ vs. normal control, ${ }^{* *} p<0.05$ vs. DOX control). MDA-malondialdehyde; SOD 1superoxide dismutase 1; SOD 2—superoxide dismutase 2; HO-1-heme oxygenase-1; GSH-reduced glutathione; Nrf 2-Nuclear factor erythroid 2-related factor 2; NOR—normal; NERO-nerolidol; DOX-doxorubicin; ND—rats administered with a single intraperitoneal dose of DOX and orally treated with NERO. 


\subsection{NERO Treatment Attenuates Induction and Release of Pro-Inflammatory Cytokines}

The levels and expressions of tumor necrosis factor- $\alpha$ (TNF- $\alpha$ ), interleukin- 6 (IL$6)$, and interleukin-1 $\beta$ (IL-1 $\beta)$ were significantly $(p<0.05)$ increased in DOX-injected rats compared to normal control rats. However, NERO treatment exhibited a significant $(p<0.05)$ reduction in the levels and expressions of TNF- $\alpha$, IL- 6 , and IL-1 $\beta$ in DOX-injected rats when compared to rats administered with just DOX (Figure 3).

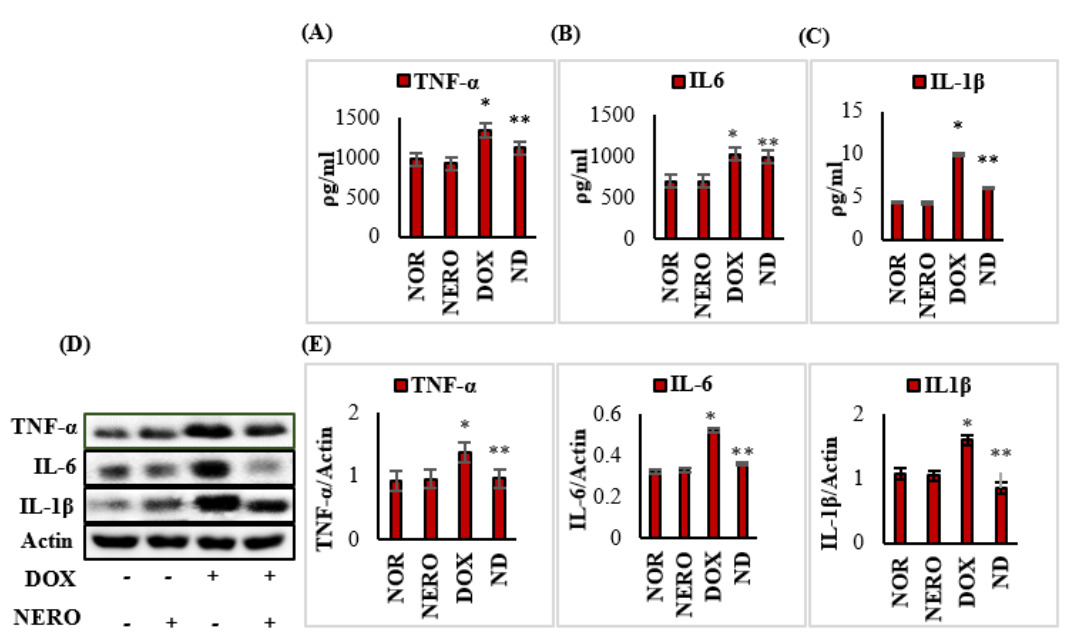

Figure 3. (A-E) Levels/expressions of TNF- $\alpha$, IL-6, and IL-1 $\beta$ in the myocardium. Each column is mean \pm SEM for eight rats in each group for ELISA and 3 rats in each group for immunoblotting. Columns do not share a common symbol $\left({ }^{*},{ }^{* *}\right)$ and differ significantly with each other $\left({ }^{*} p<\right.$ 0.05 vs. normal control, ${ }^{* *} p<0.05$ vs. DOX control). Representative images of Western immunoblot analysis and densitometric analysis for TNF- $\alpha, \mathrm{IL}-6$, and IL- $1 \beta$ in the myocardium. TNF- $\alpha$-tumor necrosis factor $\alpha$; IL-6-interleukin 6; IL-1 $\beta$-interleukin 1 $\beta$; NOR-normal; NERO-nerolidol; DOX - doxorubicin; ND—rats administered with a single intraperitoneal dose of DOX and orally treated with NERO.

3.4. NERO Attenuates Inflammatory Mediators and Modulates Altered Nuclear Factor Kappa-B $(\mathrm{NF}-\kappa \mathrm{B}) /$ Mitogen-Activated Protein Kinase (MAPK) Signaling Pathways

DOX-injected rats showed a significant $(p<0.05)$ increase in the expression of inflammatory mediators, inducible nitric oxide synthase (iNOS) and cyclooxygenase-2 (COX-2),

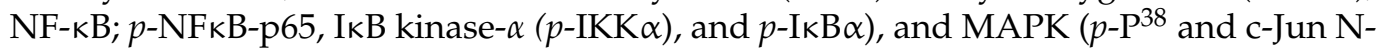
terminal kinases ( $p$-JNK) in myocardium when compared to the normal control rats. NERO treatment, however, significantly $(p<0.05)$ decreased the expression of these proteins in DOX-injected rats, at least when compared to control rats that just had DOX administered (Figure 4A,B).

\subsection{NERO Prevents DOX-Triggered DNA Damage and Apoptosis}

DOX-injected rats showed a significant $(p<0.05)$ increase in the expression of proapoptotic myocardial proteins, such as $\mathrm{H} 2 \mathrm{~A}$ histone family member $\mathrm{X}(\gamma-\mathrm{H} 2 \mathrm{AX})$, Bcl2-associatedX- protein (Bax), tumor protein (P53), apoptotic protease activating factor 1 (APAF-1), cleaved poly (ADP-ribose) polymerase (cleaved PARP), cleaved caspase-9, and cleaved caspase- 3 and cytochrome-C (Cyt-C). This was connected to a significant $(p<0.05)$ reduction in the expressions of B-cell lymphoma 2 (Bcl-2) and B-cell lymphoma-extra large (Bcl-xL) when compared to normal control rats. NERO treated rats exhibited a remarkable decrease in proapoptic proteins and induction in the expression of antiapoptotic proteins. This finding revealed its effect on cell death signaling markers in rats administered with DOX (Figure 5A,B). 


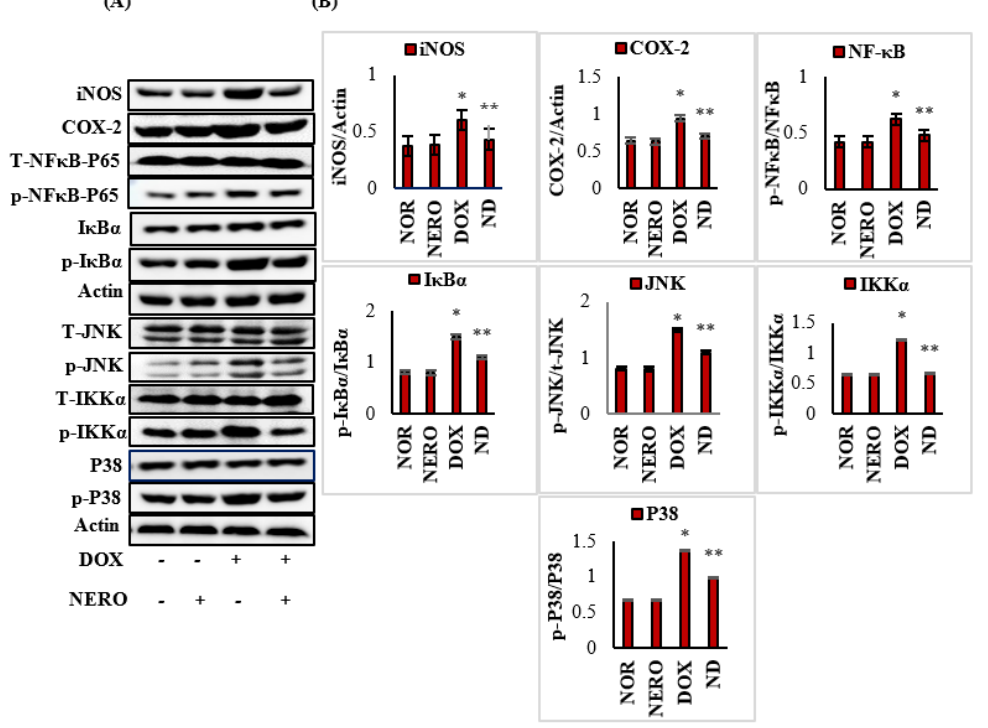

Figure 4. (A,B) Western immunoblotting and densitometric analysis for iNOS, COX-2, NF- $\mathrm{B}-\mathrm{P} 65$, $p$-NF- $\kappa \mathrm{BP} 65, \mathrm{I} \kappa \mathrm{B} \alpha$ and $p$-I $\kappa \mathrm{B} \alpha, \mathrm{t}$-IKK $\alpha, p$-IKK $\alpha, \mathrm{t}$-JNK, $p$-JNK, P38, and $p$-P38. The values are expressed as the mean $\pm \operatorname{SEM}(n=3)$; columns do not share a common symbol $(*, * *)$ and differ significantly with each other $\left({ }^{*} p<0.05\right.$ vs. normal control, ${ }^{* *} p<0.05$ vs. DOX control). iNOSinducible nitric oxide synthase; COX-2—cyclooxygenase-2; $p$-NF-kB-p65-phospho nuclear factor kappa-B-p65; t-JNK—t-Jun-amino-terminal kinase; $p$-JNK— - -Jun-amino-terminal kinase; $p$-IKK $\alpha$ IкB kinase- $\alpha$; P38-tumor protein; p-P38—phosphorilated p38; NOR-normal; NERO-nerolidol; DOX-doxorubicin; ND—rats administered with a single intraperitoneal dose of DOX and orally treated with NERO.

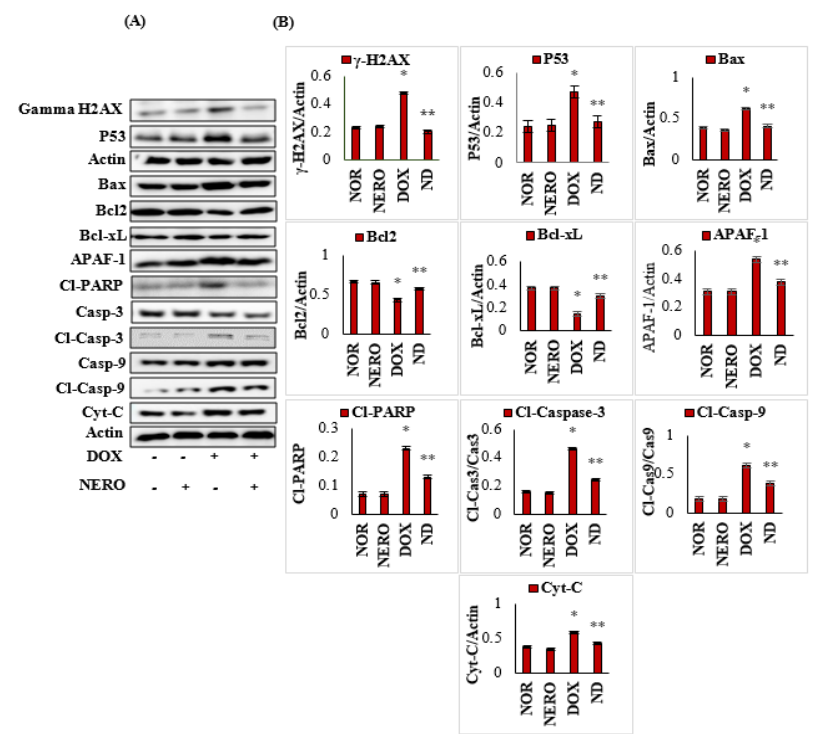

Figure 5. (A,B). Representative images of Western immunoblot analysis and densitometry for $\gamma$-H2AX, P53, Bax, Bcl2, Bcl-xL, active caspase-3, active caspase-9, APAF-1, cleaved PARP, and cytochrome-C. The values are expressed as the mean \pm SEM $(n=3)$; columns do not share a common $\operatorname{symbol}\left({ }^{*}, * *\right)$ and differ significantly with each other $\left({ }^{*} p<0.05\right.$ vs. normal control, ${ }^{* *} p<0.05$ vs. DOX control). H2AX-H2A histone family member X; Bcl-2-B-cell lymphoma 2; Bax-Bcl2-associated-Xprotein; $\mathrm{Bcl}-\mathrm{xL}$-B-cell lymphoma extra-large; P53-tumor protein; APAF-1—apoptotic protease activating factor 1; Cyt-C—cytochrome-C; CL-PARP-cleaved poly (ADP-ribose) polymerase; Casp-3caspase 3; Cl-Casp-3—cleaved caspase-3; Casp-9—caspase 9; Cl-Casp-9—cleaved caspase-9; NORnormal; NERO—nerolidol; DOX—doxorubicin; ND—rats administered with a single intraperitoneal dose of DOX and orally treated with NERO. 


\section{Discussion}

The results in the present study demonstrate the cardioprotective properties of NERO against DOX-induced acute cardiotoxicity. This was evidenced by the salvage of histopathology, restoration of myocyte injury markers and inhibition of lipid peroxidation. Further, this was substantiated by inhibition of oxidative stress, inflammation and apoptosis, mediating suppression of the Nrf2/Keap1 and NF-kB/MAPK signaling mechanisms.

Acute or chronic cardiotoxicity may appear after chemotherapy with DOX. The standard diagnostic biomarkers of myocardial cell injury in acute cardiotoxicity are troponins ( $\mathrm{T}$ and I), CK, and LDH [40,41]. A remarkable rise in these cardiac diagnostic markers in rats injected with DOX demonstrate the deleterious effects of DOX on cardiomyocytes. Interestingly, treatment with NERO appeared to protect the myocardium, as evidenced by a reduced leakage of cardiac troponins, $\mathrm{CK}$, and LDH into the circulation. The reduced leakage of myocyte injury markers concomitant to histopathological salvage of cardiomyocytes are ascribed to the potent membrane that stabilize NERO property. This fact further supports the reduction of the lipid peroxidation initiated by DOX-induced free radicals.

DOX-induced free radicals and consequent lipid peroxidation are important pathogenic events in myocardial cellular injury. The excessive generation of free radicals due to the increased oxidative metabolism and limited availability of antioxidant defense makes the myocardium more susceptible to DOX-induced cardiotoxicity [42]. The depletion of antioxidants, SOD, catalase, and GSH concomitant to lipid peroxidation after DOX injections demonstrates the occurrence of oxidative damage. Moreover, this reflects the generation of reactive oxygen species (ROS), which surpasses the antioxidant defense mechanisms [43]. Increased utilization of antioxidants for scavenging ROS might be the reason for declined antioxidant status [44]. Treatment with NERO in DOX-induced rats resulted in the normalization of SOD, catalase, and glutathione, as well as the inhibition of lipid peroxidation reflected by the reduced formation of MDA. Here, we demonstrated the antioxidant property of NERO and our findings were in agreement with previous studies wherein NERO found a well-known free radical scavenger and prevented lipid peroxidation and oxidative stress in the heart [32], liver [45] brain [31], and lungs [46].

The myocardial cells are equipped with Nrf2, a widely distributed antioxidant defense system that negatively regulates oxidative stress in tissues and curbs the occurrence of oxidative stress. In basal conditions, Nrf2, a redox-active signal protein, coexists with Keap1, a scaffold protein, for the ubiquitination and degradation of Nrf2. In cellular responses to oxidative stress, it undergoes dissociation and immediately translocates into the nucleus. This leads to the augmentation of antioxidant defense, which mediates the upregulation of detoxifying enzymes and phase II enzymes including SOD, glutathione cycle enzymes, NAD $(p)$ H: quinone oxidoreductase (NQO-1), UDP-glucuronosyl transferases (UGTs), and heme oxygenase-1 (HO-1) [47]. DOX-induced cardiotoxicity has shown the suppression or inactivation of $\mathrm{Nrf}-2$, and there is a resultant reduction in endogenous antioxidant enzyme levels and myocardial expression of HO-1. In the present study, NERO treatment significantly enhanced the activation of Nrf2/Keap1 signaling, which upregulated the endogenous antioxidant response in the myocardium. Our findings aligned with those from previous studies, wherein NERO was shown to attenuate cyclophosphamide-induced neuroinflammation, cognitive impairment [48], hepatic inflammation [45], colon inflammation [49], and acute lung-injury [50], all of which mediated Nrf2 upregulation. Regarding the interaction of NERO with the Nrf2 protein, in silico studies have shown that NERO with hydrogen bonds and hydrophobic interactions can bind the active binding site of the Nrf2 protein in its catalytic domain [48].

Myocardial inflammation is a crucial pathological alteration following DOX-chemotherapy [6]. DOX elicits inflammatory responses in myocardium by activating NF- $\mathrm{kB}$ signaling, contributing to the secretion of pro-inflammatory cytokines (IL-6, TNF- $\alpha$, and IL-1 $\beta$ ), and regulates inflammatory mediators (COX-2 and iNOS), which participates in inflammation [51,52]. NF- $\mathrm{kB}$ is a key player in the institution and propagation of inflammatory processes in an orchestrated manner, especially following the upregulation of inflammatory 
mediators and proinflammatory cytokines. The degradation of IкB $\alpha$ results in the nuclear translocation of NF- $\mathrm{kB}$, wherein it triggers the synthesis of pro-inflammatory cytokines [43]. Treatment with NERO suppressed the induction of inflammatory mediators, COX-2, and iNOS, as well as the synthesis of proinflammatory cytokines and NF- $\mathrm{kB}$, the latter of which attributes to its anti-inflammatory actions in myocardial inflammation. Additionally, in earlier studies, NERO attenuated myocardial inflammation and was induced by other chemotherapeutic drugs.

In addition to NF-kB, the MAPK family is a key regulator of signal transduction in cellular proliferation, differentiation, and apoptosis along with their primary role in regulating inflammatory mediators [53]. The JNK and p38 kinases upon inflammatory stimuli and mediator activation play critical roles in eliciting oxidative stress and inflammation in DOX-induced cardiotoxicity [16]. Considering the role of NF-kB/MAPK signaling suppression in DOX-induced cardiotoxicity, the present study investigated the ability of NERO against DOX-induced inflammatory events by assessing the components of NF$\mathrm{kB} / \mathrm{MAPK}$ signaling. In the present study, NERO treatment was observed to attenuate altered NF-kB/MAPK signaling in DOX-induced myocardial inflammation. This was concomitant to the inhibition of inflammatory mediators and histological salvages of the cardiomyocytes in DOX-challenged rats. In addition to the antioxidant properties, NERO potentially interacts with the catalytic ligand binding domain of NF- $\mathrm{kB}$ p65 by forming hydrophobic interactions to amino acid residues. This is believed to attribute to another mechanism that has an anti-inflammatory role [32]. The inhibitory action of NERO on NF- $\mathrm{KB}$ interferes with the positive feedback loop and modifies the target genes. This is the main reason for its cardioprotective effect [32].

Apart from oxidative stress and orchestrated inflammation events, apoptotic cell death of cardiomyocytes induced by DOX has been well reported in DOX-induced cardiotoxicity [54-57]. The onset and propagation of oxidative stress induced by DOX is known to elicit cardiac mitochondrial dysfunction and damage the cardiomyocytes [58]. DOX directly targets mitochondria through intrinsic apoptotic pathways and mitochondrial membrane alterations via cardiolipin binding involved in the functional changes of various mitochondrial parameters and the activities of respiratory chain enzymes [59]. Administering DOX induces protein ataxia-telangiectasia mutated (ATM) activation followed by DNA double strand breaks (DSBs), as well as the phosphorylation of p53 and H2AX [54,55]. Moreover, ATM is an apoptotic modulator during DNA damage responses (DDR) via p53 activation [60]. The myocardial protein expression of $\gamma-\mathrm{H} 2 \mathrm{AX}$ is a marker of DSBs and a primary signal for chromatin responses to cardiac DNA damage in DOX-administered rats [56,57]. The downregulation of phosphorylation of p53 and H2AX following NERO treatment indicates its potential effect on apoptotic cell death elicited by DOX in cardiomyocytes.

The onset of apoptosis is regulated by apoptotic machinery, which involves caspases (caspase-3 and 9) and members of the Bcl-2 family, which are crucial meditators of the intrinsic pathway of cell death involving pro-apoptotic signals of Bax and anti-apoptotic signals of Bcl-2 [61]. DOX-induced ROS production induces cell death by inhibiting antiapoptotic proteins namely Bcl-xL and Bcl2. Moreover, it can simultaneously promote the activation of pro-apoptotic markers [62]. Bax activation evokes apoptotic cell death by inducing mitochondrial pore formation, which then facilitates a release of cytochrome-C and cleavage of poly (ADP-ribose) polymerase. NERO treatment in DOX-injected rats downregulated Bax, active caspase-3, caspase-9, APAF-1, cleaved PARP, and cytochrome-C. Moreover, it upregulated $\mathrm{Bcl} 2$ and $\mathrm{Bcl}-\mathrm{xL}$. The downregulation of proapoptotic proteins and upregulation of antiapoptotic proteins demonstrate inhibited DOX-induced mitochondrial dysfunction, DNA damage, ATM activation, and apoptosis. This could be ascribed to its potent antioxidant property, which curbs the initiation and onset of oxidative stress in the myocardium.

Taken together, our study reveals that NERO has the potential to protect myocardium from DOX-induced acute cardiotoxicity by countering oxidative stress through enhanced Nrf2 signaling, the inhibition of inflammatory mediators and cytokines, as well as the 
amelioration of NF-kB/MAPK activation. Moreover, the study findings also demonstrate favorable modulation of apoptotic cell death pathways which supports the salvage of cardiomyocytes.

\section{Conclusions}

Our study demonstrated that NERO is a potent antioxidant that has anti-inflammatory and antiapoptotic effects in acute cardiotoxicity, especially when induced by DOX. Furthermore, we identified numerous cell signaling mechanisms involved in attributing antioxidant, anti-inflammatory, and antiapoptotic effects, all of which mediate the upregulation of Nrf2/Keap1, suppression of NF-KB/MAPK, and favorable modulation of cell death pathways. Thus, it can be concluded that NERO or plants containing NERO could be important for improving the therapeutic usefulness of DOX in chemotherapy by exerting cardioprotective effects.

Author Contributions: Conceptualization, S.O.; methodology, S.A. (Seenipandi Arunachalam), M.F.N.M.; software, M.F.N.M.; validation, M.F.N.M.; formal analysis, M.F.N.M.; investigation, M.F.N.M., S.A. (Seenipandi Arunachalam), S.A. (Sheikh Azimullah); resources, S.O.; data curation, M.F.N.M.; writing-M.F.N.M., S.A. (Seenipandi Arunachalam); writing—review and editing, S.O., S.N.G., C.S., M.F.N.M.; supervision, S.O.; project administration, S.O., M.F.N.M.; funding acquisition, S.O. All authors have read and agreed to the published version of the manuscript.

Funding: The authors sincerely acknowledge the research grant support from College of Medicine and Health Sciences, UAE University, Al Ain, UAE.

Institutional Review Board Statement: The protocols and procedures for animal experiments and research were approved by the Animal Ethics Committee of the United Arab Emirates University, Al Ain, UAE (ethical code: ERA_2019_5919). The rats were obtained from the University Animal Research Facility of the College of Medicine and Health Sciences (CMHS), United Arab Emirates University, Al Ain, UAE.

Informed Consent Statement: Not applicable.

Data Availability Statement: The data presented in the present study will be available from the corresponding author upon reasonable request.

Conflicts of Interest: The authors have no competing interests to declare. The funders of the research grant have no role in study design, interpretation, and publication. The received funding did not lead to any conflict of interests regarding the publication of this manuscript.

\section{References}

1. Parent, S.; Pituskin, E.; Paterson, D.I. The cardio-oncology program: A multidisciplinary approach to the care of cancer patients with cardiovascular disease. Can. J. Cardiol. 2016, 32, 847-851. [CrossRef]

2. Weiss, R.B. The anthracyclines: Will we ever find a better doxorubicin? Semin. Oncol. 1992, 19, 670-686.

3. Carvalho, C.; Santos, R.X.; Cardoso, S.; Correia, S.; Oliveira, P.J.; Santos, M.S.; Moreira, P.I. Doxorubicin: The good, the bad and the ugly effect. Curr. Med. Chem. 2009, 16, 3267-3285. [CrossRef]

4. Trapani, D.; Zagami, P.; Nicolò, E.; Pravettoni, G.; Curigliano, G. Management of cardiac toxicity induced by chemotherapy. J. Clin. Med. 2020, 9, 2885. [CrossRef]

5. Zhang, J.; Wang, M.; Ding, W.; Zhao, M.; Ye, J.; Xu, Y.; Wang, Z.; Ye, D.; Li, D.; Liu, J. Resolvin E1 protects against doxorubicin-induced cardiotoxicity by inhibiting oxidative stress, autophagy and apoptosis by targeting AKT/mTOR signaling. Biochem. Pharmacol. 2020, 180, 114188. [CrossRef] [PubMed]

6. Al-Taee, H.; Azimullah, S.; Meeran, M.F.N.; Almheiri, M.K.A.; Jasmi, R.A.A.; Tariq, S.; Khan, M.A.B.; Adeghate, E.; Ojha, S. $\beta$ caryophyllene, a dietary phytocannabinoid attenuates oxidative stress, inflammation, apoptosis and prevents structural alterations of the myocardium against doxorubicin-induced acute cardiotoxicity in rats: An in vitro and in vivo study. Eur. J. Pharmacol. 2019, 858, 172467. [CrossRef] [PubMed]

7. Reichardt, P.; Tabone, M.-D.; Mora, J.; Morland, B.; Jones, R.L. Risk-benefit of dexrazoxane for preventing anthracycline-related cardiotoxicity: Re-evaluating the European labeling. Future Oncol. 2018, 14, 2663-2676. [CrossRef]

8. Shah, S.M.A.; Akram, M.; Riaz, M.; Munir, N.; Rasool, G. Cardioprotective potential of plant-derived molecules: A scientific and medicinal approach. Dose-Response 2019, 17. [CrossRef] [PubMed]

9. Ojha, S.; Taee, H.A.; Goyal, S.; Mahajan, U.B.; Patil, C.R.; Arya, D.S.; Rajesh, M. Cardioprotective potentials of plant-derived small molecules against doxorubicin associated cardiotoxicity. Oxidative Med. Cell. Longev. 2016, 2016. [CrossRef] [PubMed] 
10. Yu, J.; Wang, C.; Kong, Q.; Wu, X.; Lu, J.-J.; Chen, X. Recent progress in doxorubicin-induced cardiotoxicity and protective potential of natural products. Phytomedicine 2018, 40, 125-139. [CrossRef] [PubMed]

11. Kamble, S.M.; Goyal, S.N.; Patil, C.R. Multifunctional pentacyclic triterpenoids as adjuvants in cancer chemotherapy: A review. RSC Adv. 2014, 4, 33370-33382. [CrossRef]

12. Van der Zanden, S.Y.; Qiao, X.; Neefjes, J. New insights into the activities and toxicities of the old anticancer drug doxorubicin. FEBS J. 2020. [CrossRef] [PubMed]

13. Minotti, G.; Menna, P.; Salvatorelli, E.; Cairo, G.; Gianni, L. Anthracyclines: Molecular advances and pharmacologic developments in antitumor activity and cardiotoxicity. Pharmacol. Rev. 2004, 56, 185-229. [CrossRef] [PubMed]

14. Meeran, M.F.N.; Taee, H.A.; Azimullah, S.; Tariq, S.; Adeghate, E.; Ojha, S. $\beta$-Caryophyllene, a natural bicyclic sesquiterpene attenuates doxorubicin-induced chronic cardiotoxicity via activation of myocardial cannabinoid type-2 (CB2) receptors in rats. Chem. Biol. Interact. 2019, 304, 158-167. [CrossRef]

15. Sahu, R.; Dua, T.K.; Das, S.; Feo, V.D.; Dewanjee, S. Wheat phenolics suppress doxorubicin-induced cardiotoxicity via inhibition of oxidative stress, MAP kinase activation, NF-kB pathway, PI3K/Akt/mTOR impairment, and cardiac apoptosis. Food Chem. Toxicol. 2019, 125, 503-519. [CrossRef]

16. Das, J.; Ghosh, J.; Manna, P.; Sil, P.C. Taurine suppresses doxorubicin-triggered oxidative stress and cardiac apoptosis in rat via up-regulation of PI3-K/Akt and inhibition of p53, p38-JNK. Biochem. Pharmacol. 2011, 81, 891-909. [CrossRef]

17. Edwardson, D.W.; Narendrula, R.; Chewchuk, S.; Mispel-Beyer, K.; Mapletoft, J.P.; Parissenti, A.M. Role of drug metabolism in the cytotoxicity and clinical efficacy of anthracyclines. Curr. Drug Metab. 2015, 16, 412-426. [CrossRef]

18. Qi, W.; Boliang, W.; Xiaoxi, T.; Guoqiang, F.; Jianbo, X.; Gang, W. Cardamonin protects against doxorubicin-induced cardiotoxicity in mice by restraining oxidative stress and inflammation associated with Nrf2 signaling. Biomed. Pharmacother. 2020, $122,109547$. [CrossRef] [PubMed]

19. Yarmohammadi, F.; Rezaee, R.; Haye, A.W.; Karimi, G. Endoplasmic reticulum stress in doxorubicin-induced cardiotoxicity may be therapeutically targeted by natural and chemical compounds: A review. Pharmacol. Res. 2021, 164, 105383. [CrossRef]

20. Xu, Z.-M.; Li, C.-B.; Liu, Q.-L.; Li, P.; Yang, H. Ginsenoside Rg1 prevents doxorubicin-induced cardiotoxicity through the inhibition of autophagy and endoplasmic reticulum stress in mice. Int. J. Mol. Sci. 2018, 19, 3658. [CrossRef] [PubMed]

21. Yu, J.; Gao, H.; Wu, C.; Xu, Q.-M.; Lu, J.-J.; Chen, X. Diethyl blechnic, a novel natural product isolated from Salvia miltiorrhiza Bunge, inhibits doxorubicin-induced apoptosis by inhibiting ROS and activating JNK1/2. Int. J. Mol. Sci. 2018, 19, 1809. [CrossRef]

22. Alanazi, A.M.; Fadda, L.; Alhusaini, A.; Ahmad, R.; Hasan, I.H.; Mahmoud, A.M. Liposomal resveratrol and/or carvedilol attenuate doxorubicin-induced cardiotoxicity by modulating inflammation, oxidative stress and S100A1 in rats. Antioxidants 2020, 9, 159. [CrossRef]

23. Chan, W.K.; Tan, L.T.; Chan, K.G.; Lee, L.H.; Goh, B.H. Nerolidol: A sesquiterpene alcohol with multi-faceted pharmacological and biological activities. Molecules 2016, 21, 529. [CrossRef]

24. Zhou, Y.; Zeng, L.; Liu, X.; Gui, J.; Mei, X.; Fu, X.; Dong, F.; Tang, J.; Zhang, L.; Yang, Z. Formation of (E)-nerolidol in tea (Camellia sinensis) leaves exposed to multiple stresses during tea manufacturing. Food Chem. 2017, 231, 78-86. [CrossRef] [PubMed]

25. Alves, R.F.; Nascimento, A.M.D.; Nogueira, J.M.F. Characterization of the aroma profile of Madeira wine by sorptive extraction techniques. Anal. Chim. Acta 2005, 546, 11-21. [CrossRef] [PubMed]

26. Green, S.A.; Chen, X.; Nieuwenhuizen, N.J.; Matich, A.J.; Wang, M.Y.; Bunn, B.J.; Yauk, Y.-K.; Atkinson, R.G. Identification, functional characterization, and regulation of the enzyme responsible for floral (E)-nerolidol biosynthesis in kiwifruit (Actinidia chinensis). J. Exp. Bot. 2012, 63, 1951-1967. [CrossRef] [PubMed]

27. Yan, J.W.; Ban, Z.J.; Lu, H.Y.; Li, D.; Poverenov, E.; Luo, Z.S.; Li, L. The aroma volatile repertoire in strawberry fruit: A review. J. Sci. Food Agric. 2018, 98, 4395-4402. [CrossRef] [PubMed]

28. Carvalho, R.B.F.D.; Almeida, A.A.C.D.; Campelo, N.B.; Lellis, D.R.O.D.; Nunes, L.C.C. Nerolidol and its pharmacological application in treating neurodegenerative diseases: A review. Recent Pat. Biotechnol. 2018, 12, 158-168. [CrossRef]

29. Zhou, M.-X.; Li, G.-H.; Sun, B.; Xu, Y.-W.; Li, A.-L.; Li, Y.-R.; Ren, D.-M.; Wang, X.-N.; Wen, X.-S.; Lou, H.-X. Identification of novel Nrf2 activators from Cinnamomum chartophyllum $\mathrm{HW} \mathrm{Li}$ and their potential application of preventing oxidative insults in human lung epithelial cells. Redox Biol. 2018, 14, 154-163. [CrossRef] [PubMed]

30. Fonsêca, D.V.; Salgado, P.R.R.; Carvalho, F.L.D.; Salvadori, M.G.S.S.; Penha, A.R.S.; Leite, F.C.; Borges, C.J.S.; Piuvezam, M.R.; Pordeus, L.C.D.M.; Sousa, D.P. Nerolidol exhibits antinociceptive and anti-inflammatory activity: Involvement of the GABA ergic system and proinflammatory cytokines. Fundam. Clin. Pharmacol. 2016, 30, 14-22. [CrossRef]

31. Javed, H.; Azimullah, S.; Khair, S.B.A.; Ojha, S.; Haque, M.E. Neuroprotective effect of nerolidol against neuroinflammation and oxidative stress induced by rotenone. BMC Neurosci. 2016, 17, 58. [CrossRef] [PubMed]

32. Iqubal, A.; Sharma, S.; Ansari, M.A.; Najmi, A.K.; Syed, M.A.; Ali, J.; Alam, M.M.; Ahmad, S.; Haque, S.E. Nerolidol attenuates cyclophosphamide-induced cardiac inflammation, apoptosis and fibrosis in Swiss Albino mice. Eur. J. Pharmacol. 2019, 863, 172666. [CrossRef] [PubMed]

33. Asaikumar, L.; Vennila, L.; Akila, P.; Sivasangari, S.; Kanimozhi, K.; Premalatha, V.; Sindhu, G. Expression of concern: Preventive effect of nerolidol on isoproterenol induced myocardial damage in Wistar rats: Evidences from biochemical and histopathological studies. Drug Dev. Res. 2019, 80, 814-823. [CrossRef] [PubMed] 
34. Vasconcelos, C.M.L.; Oliveira, I.S.N.; Santos, J.N.A.; Souza, A.A.; Menezes-Filho, J.E.R.; Neto, J.A.S.; Lima, T.C.; Sousa, D.P.D. Negative inotropism of terpenes on guinea pig left atrium: Structure-activity relationships. Nat. Prod. Res. 2018, 32, $1428-1431$. [CrossRef] [PubMed]

35. Biazi, B.I.; Zanetti, T.A.; Baranoski, A.; Corveloni, A.C.; Mantovani, M.S. Cis-nerolidol induces endoplasmic reticulum stress and cell death in human hepatocellular carcinoma cells through extensive CYP2C19 and CYP1A2 oxidation. Basic Clin. Pharmacol. Toxicol. 2017, 121, 334-341. [CrossRef]

36. Hanušová, V.; Caltová, K.; Svobodová, H.; Ambrož, M.; Skarka, A.; Murínová, N.; Králová, V.; Tomšík, P.; Skálová, L. The effects of $\beta$-caryophyllene oxide and trans-nerolidol on the efficacy of doxorubicin in breast cancer cells and breast tumor-bearing mice. Biomed. Pharmacother. 2017, 95, 828-836. [CrossRef]

37. Ambrož, M.; Boušová, I.; Skarka, A.; Hanušová, V.; Králová, V.; Matoušková, P.; Szotáková, B.; Skálová, L. The influence of sesquiterpenes from Myrica rubra on the antiproliferative and pro-oxidative effects of doxorubicin and its accumulation in cancer cells. Molecules 2015, 20, 15343-15358. [CrossRef] [PubMed]

38. Wattenberg, L.W. Inhibition of azoxymethane-induced neoplasia of the large bowel by 3-hydroxy-3, 7, 11-trimethyl-1, 6, 10dodecatriene (nerolidol). Carcinogenesis 1991, 12, 151-152. [CrossRef] [PubMed]

39. Ambrož, M.; Matoušková, P.; Skarka, A.; Zajdlová, M.; Žáková, K.; Skálová, L. The effects of selected sesquiterpenes from Myrica rubra essential oil on the efficacy of doxorubicin in sensitive and resistant cancer cell lines. Molecules 2017, 22, 1021. [CrossRef]

40. Meeran, M.F.N.; Jagadeesh, G.S.; Selvaraj, P. Thymol attenuates altered lipid metabolism in $\beta$-adrenergic agonist induced myocardial infarcted rats by inhibiting tachycardia, altered electrocardiogram, apoptosis and cardiac hypertrophy. J. Funct. Foods 2015, 14, 51-62. [CrossRef]

41. Jagadeesh, G.S.; Meeran, M.F.N.; Selvaraj, P. Protective effects of 7-hydroxycoumarin on dyslipidemia and cardiac hypertrophy in isoproterenol-induced myocardial infarction in rats. J. Biochem. Mol. Toxicol. 2016, 30, 120-127. [CrossRef]

42. Sahu, B.D.; Kumar, J.M.; Kuncha, M.; Borkar, R.M.; Srinivas, R.; Sistla, R. Baicalein alleviates doxorubicin-induced cardiotoxicity via suppression of myocardial oxidative stress and apoptosis in mice. Life Sci. 2016, 144, 8-18. [CrossRef] [PubMed]

43. Wang, S.; Wang, Y.; Zhang, Z.; Liu, Q.; Gu, J. Cardioprotective effects of fibroblast growth factor 21 against doxorubicin-induced toxicity via the SIRT1/LKB1/AMPK pathway. Cell Death Dis. 2017, 8, e3018. [CrossRef] [PubMed]

44. Mantawy, E.M.; El-Bakly, W.M.; Esmat, A.; Badr, A.M.; El-Demerdash, E. Chrysin alleviates acute doxorubicin cardiotoxicity in rats via suppression of oxidative stress, inflammation and apoptosis. Eur. J. Pharmacol. 2014, 728, 107-118. [CrossRef] [PubMed]

45. Iqubal, A.; Syed, M.A.; Ali, J.; Najmi, A.K.; Haque, M.M.; Haque, S.E. Nerolidol protects the liver against cyclophosphamideinduced hepatic inflammation, apoptosis, and fibrosis via modulation of Nrf2, NF- $\mathrm{B}$ p 65 , and caspase-3 signaling molecules in Swiss albino mice. BioFactors 2020. [CrossRef]

46. Leung, W.-S.; Yang, M.-L.; Lee, S.-S.; Kuo, C.-W.; Ho, Y.-C.; Huang-Liu, R.; Lin, H.-W.; Kuan, Y.-H. Protective effect of zerumbone reduces lipopolysaccharide-induced acute lung injury via antioxidative enzymes and Nrf2/HO-1 pathway. Int. Immunopharmacol. 2017, 46, 194-200. [CrossRef] [PubMed]

47. Baiyun, R.; Li, S.; Liu, B.; Lu, J.; Lv, Y.; Xu, J.; Wu, J.; Li, J.; Lv, Z.; Zhang, Z. Luteolin-mediated PI3K/AKT/Nrf2 signaling pathway ameliorates inorganic mercury-induced cardiac injury. Ecotoxicol. Environ. Saf. 2018, 161, 655-661. [CrossRef] [PubMed]

48. Iqubal, A.; Sharma, S.; Najmi, A.K.; Syed, M.A.; Ali, J.; Alam, M.M.; Haque, S.E. Nerolidol ameliorates cyclophosphamide-induced oxidative stress, neuroinflammation and cognitive dysfunction: Plausible role of Nrf2 and NF-кB. Life Sci. 2019, $236,116867$. [CrossRef]

49. Raj, V.; Venkataraman, B.; Almarzooqi, S.; Chandran, S.; Ojha, S.K.; Attoub, S.; Adrian, T.E.; Subramanya, S.B. Nerolidol mitigates colonic inflammation: An experimental study using both in vivo and in vitro models. Nutrients 2020, 12, 2032. [CrossRef]

50. Ni, Y.-L.; Shen, H.-T.; Su, C.-H.; Chen, W.-Y.; Huang-Liu, R.; Chen, C.-J.; Chen, S.-P.; Kuan, Y.-H. Nerolidol suppresses the inflammatory response during lipopolysaccharide-induced acute lung injury via the modulation of antioxidant enzymes and the AMPK/Nrf-2/HO-1 pathway. Oxidative Med. Cell. Longev. 2019, 2019. [CrossRef]

51. Yang, J.; Wang, Z.; Chen, D.-L. Shikonin ameliorates isoproterenol (ISO)-induced myocardial damage through suppressing fibrosis, inflammation, apoptosis and ER stress. Biomed. Pharmacother. 2017, 93, 1343-1357. [CrossRef]

52. Kim, D.H.; Chung, J.H.; Yoon, J.S.; Ha, Y.M.; Bae, S.; Lee, E.K.; Jung, K.J.; Kim, M.S.; Kim, Y.J.; Kim, M.K. Ginsenoside Rd inhibits the expressions of iNOS and COX-2 by suppressing NF- KB in LPS-stimulated RAW264. 7 cells and mouse liver. J. Ginseng Res. 2013, 37, 54. [CrossRef]

53. El-Agamy, D.S.; El-Harbi, K.M.; Khoshhal, S.; Ahmed, N.; Elkablawy, M.A.; Shaaban, A.A.; Abo-Haded, H.M. Pristimerin protects against doxorubicin-induced cardiotoxicity and fibrosis through modulation of Nrf2 and MAPK/NF-kB signaling pathways. Cancer Manag. Res. 2019, 11, 47. [CrossRef]

54. Burma, S.; Chen, B.P.; Murphy, M.; Kurimasa, A.; Chen, D.J. ATM phosphorylates histone H2AX in response to DNA doublestrand breaks. J. Biol. Chem. 2001, 276, 42462-42467. [CrossRef]

55. Banin, S.; Moyal, L.; Shieh, S.Y.; Taya, Y.; Anderson, C.W.; Chessa, L.; Smorodinsky, N.I.; Prives, C.; Reiss, Y.; Shiloh, Y. Enhanced phosphorylation of p53 by ATM in response to DNA damage. Science 1998, 281, 1674-1677. [CrossRef]

56. Siddiqui, M.S.; François, M.; Fenech, M.F.; Leifert, W.R. Persistent $\gamma$ H2AX: A promising molecular marker of DNA damage and aging. Mutat. Res. Rev. Mutat. Res. 2015, 766, 1-19. [CrossRef] [PubMed]

57. Xu, Y.; Price, B.D. Chromatin dynamics and the repair of DNA double strand breaks. Cell Cycle 2011, 10, 261-267. [CrossRef] [PubMed] 
58. Huang, L.; Zhang, K.; Guo, Y.; Huang, F.; Yang, K.; Chen, L.; Huang, K.; Zhang, F.; Long, Q.; Yang, Q. Honokiol protects against doxorubicin cardiotoxicity via improving mitochondrial function in mouse hearts. Sci. Rep. 2017, 7, 1-12. [CrossRef] [PubMed]

59. Kuznetsov, A.V.; Margreiter, R.; Amberger, A.; Saks, V.; Grimm, M. Changes in mitochondrial redox state, membrane potential and calcium precede mitochondrial dysfunction in doxorubicin-induced cell death. Biochim. Biophys. Acta Mol. Cell Res. 2011, 1813, 1144-1152. [CrossRef]

60. Shiloh, Y.; Ziv, Y. The ATM protein kinase: Regulating the cellular response to genotoxic stress, and more. Nat. Rev. Mol. Cell Biol. 2013, 14, 197-210. [CrossRef]

61. Radhiga, T.; Rajamanickam, C.; Sundaresan, A.; Ezhumalai, M.; Pugalendi, K.V. Effect of ursolic acid treatment on apoptosis and DNA damage in isoproterenol-induced myocardial infarction. Biochimie 2012, 94, 1135-1142. [CrossRef] [PubMed]

62. Redza-Dutordoir, M.; Averill-Bates, D.A. Activation of apoptosis signalling pathways by reactive oxygen species. Biochim. Biophys. Acta Mol. Cell Res. 2016, 1863, 2977-2992. [CrossRef] [PubMed] 